\title{
Solitary Metastasis of Gastric Carcinoma to the Cerebellopontine Angle
}

\author{
Dong-Wook Lim ${ }^{1}$, Eun-Young Kim², Kyu-Sung Kim ${ }^{1}$ and Ho-Seok Choi ${ }^{3}$ \\ ${ }^{1}$ Departments of Otorhinolaryngology, ${ }^{2}$ Neurosurgery, Inha University Hospital, Incheon, \\ ${ }^{3}$ Department of Otorhinolaryngology, Soonchunhyang University Hospital, Gumi, Korea
}

\section{Received June 18, 2013 \\ Revised August 9, 2013 \\ Accepted August 15, 2013}

Internal auditory canal (IAC) and cerebellopontine metastases are very rare lesions and their diagnosis is very difficult due to their similarity to vestibular schwannoma in clinical characteristics and radiologic findings. Our case is peculiar and differs from previously reported cases of malignant metastasis to the IAC in two aspects: 1) solitary IAC metastasis occurred without distant metastasis and 2) symptoms due to IAC metastasis preceded outbreak of primary lesion symptoms or diagnosis of primary lesion. In our literature search, no correlating cases have been reported. In this peculiar case, rapid progression of otologic symptoms, short duration of onset from hearing impairment to facial palsy, and laterally eccentric contrast enhancement in gadolinium-enhanced T1-weighted magnetic resonance imaging may have been clues for metastatic lesion.

Korean J Audiol 2013;17:94-96

\section{Introduction}

The most common tumor occupying internal auditory canal (IAC) and cerebellopontine angle is vestibular schwannoma, followed by meningioma, epidermoid cyst, glomus jugulare tumors, and facial and trigeminal neuroma. ${ }^{1)}$ Metastatic carcinoma found in IAC or cerebellopontine angle is an extremely uncommon entity with a very low incidence. ${ }^{2)}$ Rapid progression of symptoms, short duration of onset from hearing impairment to facial palsy, and laterally eccentric contrast enhancement in gadolinium-enhanced T1-weighted magnetic resonance imaging (MRI) are the clues for metastatic lesion. There was no report found in our literature search concerning solitary metastasis of gastric carcinoma to IAC without presenting any gastric symptoms, therefore we report this case of metastatic carcinoma in IAC removed by translabyrithine approach.

\section{Case Report}

Institutional Review Board (IRB) approval from IRB committee of Inha university hospital and informed consent were obtained before chart review (IRB number: 13-0106). A 59-year-old man was referred to our clinic with a week of leftsided facial paralysis (House-Brackmann grade III) accompanied by one month's history of left-sided hearing loss. The patient did not have any symptoms of dizziness or observable spontaneous nystagmus. His pure tone audiogram demonstrated a total loss of hearing on the left side and normal hearing on the right side. Vestibular evoked myogenic potential test revealed loss of typical waveforms throughout all frequencies on the left side. On his temporal bone computed tomography, no pathological findings were identified. T1-weighted MRI scan with gadolinium contrast showed an enhancing mass filling the whole left IAC and extending rather slightly laterally eccentric into cerebellopontine angle (Fig. 1). The same lesion was observed as an iso-intense signal on T2 sequence.

Under provisional diagnosis of vestibular schwannoma with possibility of metastatic lesion, tumor removal was performed by translabyrinthine approach. The mass encasing the facial nerve was a rather brownish soft mass severely adhered to the acousticofacial bundle from the porus throughout the labyrinthine segment of the facial nerve (Fig. 2). Due to severe adhesion and possible neural invasion on frozen biopsy, the facial nerve was sacrificed from the porus to laby- 


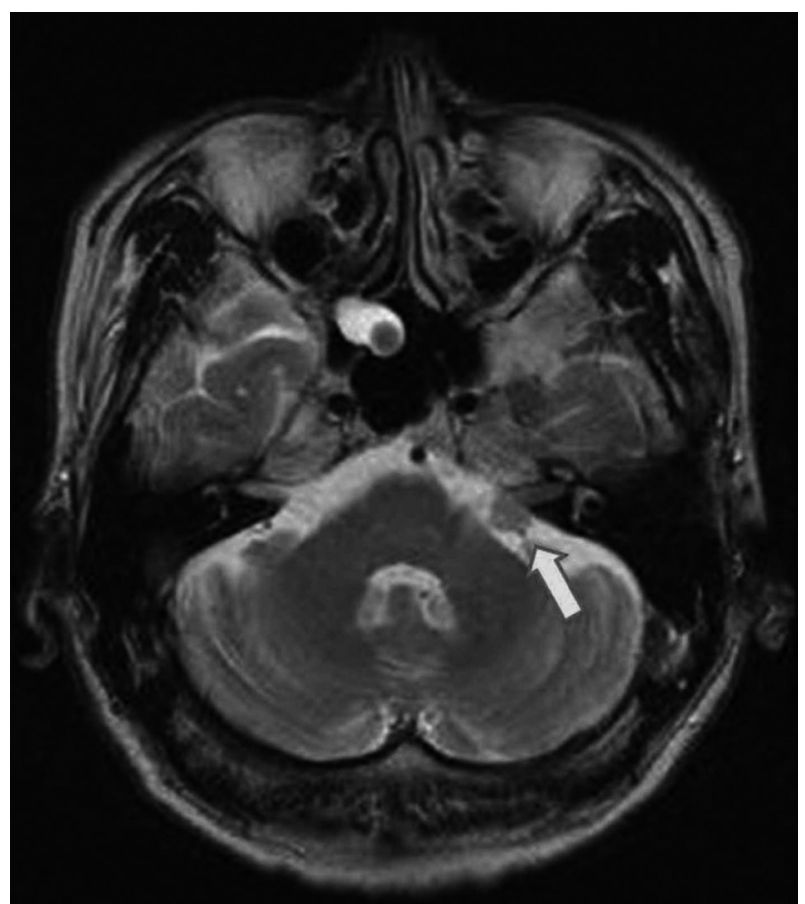

Fig. 1. T1-weighted MRI scan with gadolinium contrast showing an enhancing mass filling the whole left IAC and extending rather slightly laterally eccentric into cerebellopontine angle (arrow). MRI: magnetic resonance imaging, IAC: internal auditory canal.

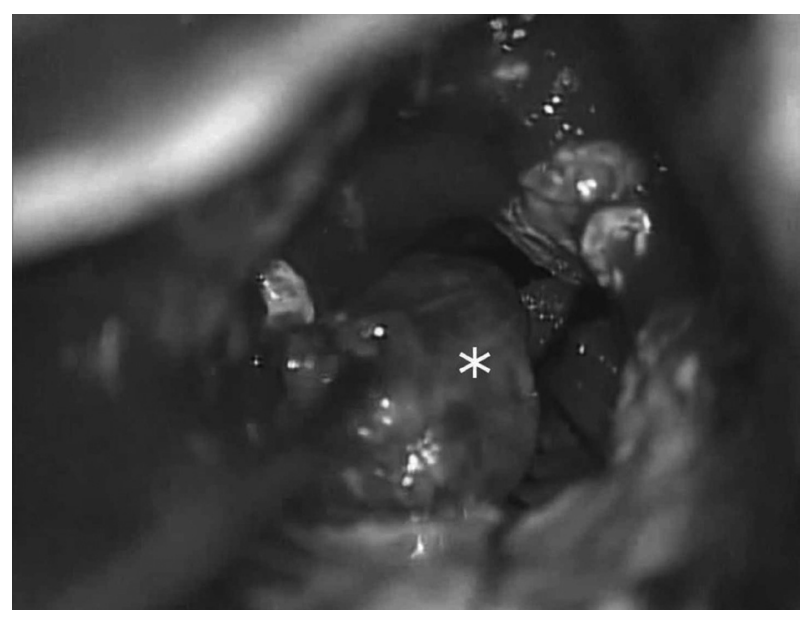

Fig. 2. Mass encasing the facial nerve presenting a rather brownish soft mass severely adhered to the acousticofacial bundle from the porus throughout the labyrinthine segment of the facial nerve (asterisk).

rinthine segment together with the tumor mass. Nerve reconstruction was performed with the sural nerve. Histopathological study revealed a poorly cohesive adenocarcinoma with intraneural invasion.

Positron emission tomography scan revealed high uptake in the gastric antrum, endoscopic study showed a fungating mass on distal antrum, and biopsy confirmed a poorly cohesive adenocarcinoma that matched the pathologic result for the IAC mass.
The follow-up MRI revealed no significant sign of residual lesion, and the patient was transferred to the general surgery department for subtotal gastrectomy. After gastric surgery, he is receiving palliative chemotherapy through the oncology department. The facial palsy aggravated to complete paralysis and is now being managed conservatively.

\section{Discussion}

Metastatic carcinoma found in IAC or cerebellopontine angle is an extremely uncommon entity with an incidence of $0.2 \%$ to $0.7 \%$ of all cerebellopontine angle tumors, which are also rare. ${ }^{1)}$ Furthermore, isolated metastasis of primary gastric adenocarcinoma without other metastatic lesion or any medical history of primary lesion is exceedingly rare; in fact, no reported case was found. The most common primary malignancies that metastasize to the IAC are breast, lung, prostate, kidney, and colon. ${ }^{1,2)}$

Considering that most tumors of IAC are slow-growing, the most distinct clinical feature that should alert otoneurosurgeons is rapid progression and short interval between the onset of hearing loss and facial palsy. The reason for this rapid clinical course is due to the rapid growth of the metastatic carcinoma. $^{2,3)}$

MRI findings of metastatic lesion might provide some clues such as bilateral involvement or thick linear and extra-axial contrast enhancement in Gadolinium enhanced T1-weighted images. ${ }^{1,4)}$ Lumbar puncture can aid the diagnostic process by giving additional information preoperatively. ${ }^{5)}$

The prognosis of all cases reported by Preuss, et al. ${ }^{5)}$ was very poor. The average survival was only 6 weeks after diagnosis in untreated patients and 12 weeks in treated patients. Prognosis was improved by the combination of complete surgical removal and radiation therapy.

Management of patients with malignant metastasis to the cerebellopontine angle attempts to prevent further deterioration of hearing loss and neurologic deficit. ${ }^{5)}$ Once metastasis is suspected, a multidisciplinary approach of treatment including surgical removal (or stereotactic radiosurgery), chemotherapy, and radiotherapy is recommended. ${ }^{1)}$ In our case, the lesion was removed via translabyrinthine approach and chemotherapy was planned. However, the decision on radiotherapy was reserved since biopsy revealed a poorly cohesive adenocarcinoma so that a palliative approach was preferred.

\section{Acknowledgments}

This work was supported in part by the Soonchunhyang University Research Fund. 


\section{REFERENCES}

1) Chang KH, Song CE, Seo JH, Yeo SW. Solitary metastasis of bronchogenic adenocarcinoma to the internal auditory canal: a case report. J Korean Med Sci 2009;24:1227-9.

2) Falcioni M, Piccirillo E, Di Trapani G, Romano G, Russo A. Internal auditory canal metastasis. Acta Otorhinolaryngol Ital 2004;24:7882.

3) Han JK, Park KH, Lee MS, Lee CK. Gastric carcinoma metastasis to the internal auditory canal. Otol Neurotol 2012;33:e19-20.

4) Rohlfs AK, Burger R, Viebahn C, Held P, Woenckhaus M, Römer $\mathrm{FW}$, et al. Uncommon lesions in the internal auditory canal (IAC): review of the literature and case report. J Neurol Surg A Cent Eur Neurosurg 2012;73:160-6.

5) Preuss M, Stein M, Huegens-Penzel M, Kuchelmeister K, Nestler U. Metastatic tumours mimicking vestibular schwannoma. Acta Neurochir (Wien) 2008;150:915-9. 